\title{
A local constitutive model with anisotropy for ratcheting under 2D axial-symmetric isobaric deformation
}

\author{
V. Magnanimo $\cdot$ S. Luding
}

Received: 28 October 2010 / Published online: 12 May 2011

(C) The Author(s) 2011. This article is published with open access at Springerlink.com

\begin{abstract}
A local constitutive model for anisotropic granular materials is introduced and applied to isobaric (homogeneous) axial-symmetric deformation. The simplified model (in the coordinate system of the bi-axial box) involves only scalar values for hydrostatic and shear stresses, for the volumetric and shear strains as well as for the new ingredient, the anisotropy modulus. The non-linear constitutive evolution equations that relate stress and anisotropy to strain are inspired by observations from discrete element method (DEM) simulations. For the sake of simplicity, parameters like the bulk and shear modulus are set to constants, while the shear stress ratio and the anisotropy evolve with different rates to their critical state limit values when shear deformations become large. When applied to isobaric deformation in the bi-axial geometry, the model shows ratcheting under cyclic loading. Fast and slow evolution of the anisotropy modulus with strain. Lead to dilatancy and contractancy, respectively. Furthermore, anisotropy acts such that it works "against" the strain/stress, e.g., a compressive strain builds up anisotropy that creates additional stress acting against further compression.
\end{abstract}

Keywords Constitutive modeling with anisotropy - Biaxial box $\cdot$ Ratcheting $\cdot$ Isobaric cyclic loading

\section{Introduction}

Dense granular materials show interesting behavior and special properties, different from classical fluids or solids

V. Magnanimo $(\varangle) \cdot$ S. Luding

Multi Scale Mechanics (MSM), CTW, UTwente,

PO Box 217, 7500 AE Enschede, Netherlands

e-mail:v.magnanimo@utwente.nl

S. Luding

e-mail: s.luding@utwente.nl
[5,9]. These involve dilatancy, yield stress, history dependence, as well as ratcheting $[1,2]$ and anisotropy $[6,13,22-$ 24] — among many others.

If an isotropic granular packing is subject to isotropic compression the shear stress remains close to zero and the isotropic stress can be related to the volume fraction [8]. Under shear deformation, the shear stress builds up until it reaches a yield-limit, as described by classical and more recent models, e.g. [11,13,23,24]. Also the anisotropy of the contact network varies, as related to the opening and closing of contacts, restructuring, and the creation and destruction of force-chains, as confirmed by DEM simulations [15,25]. This is at the origin of the interesting behavior of granular media, but is neglected in many continuum models of particulate matter. Only few theories, see e.g. [2,7,15,18-20,22] and references therein, involve an anisotropy state variable. The influence of the micromechanics on the non-coaxiality of stress, strain and anisotropy of soils is described e.g. in [24]. This is an essential part of a constitutive model for granular matter because it contains the information how the different modes of deformation have affected the mechanical state of the system. In this sense, anisotropy is a history variable.

In the following, a recently proposed constitutive model [17] is briefly presented and then applied to isobaric axialsymmetric deformation. The (classical) bulk and shear moduli are constants here, in order to be able to focus on the effect of anisotropy and the anisotropy related material parameters exclusively.

\section{Model system}

In order to keep the model as simple as possible, we restrict ourselves to bi-axial deformations and the bi-axial 


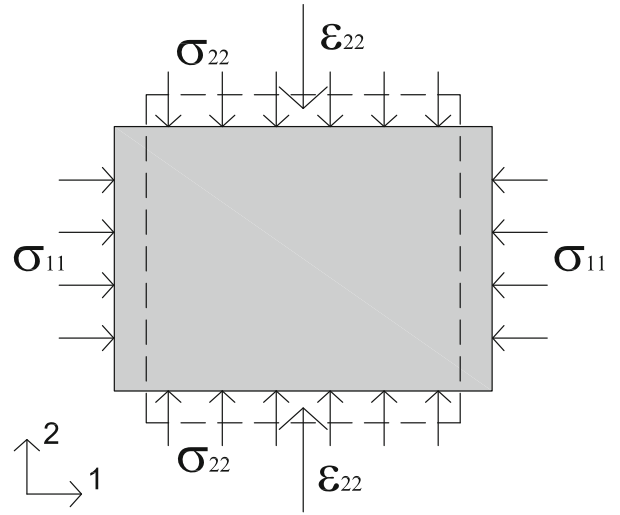

Fig. 1 Illustration of the bi-axial model system with prescribed vertical displacement $\varepsilon_{22}(t)<0$ and constant isotropic confining stress $\sigma^{h}=\left(\sigma_{11}+\sigma_{22}\right) / 2=$ const. $=\sigma_{0}<0$

orientation of the coordinate system. The bi-axial box is shown schematically in Fig. 1, where the strain in the $2-$ direction is prescribed. Due to this geometry (assuming perfectly smooth walls), in the global coordinate system one has the strain and the stress with only diagonal components that, naturally, are the eigenvalues of the two coaxial tensors:

$[\boldsymbol{\varepsilon}]=\left[\begin{array}{ll}\varepsilon_{11} & 0 \\ 0 & \varepsilon_{22}\end{array}\right], \quad[\boldsymbol{\sigma}]=\left[\begin{array}{ll}\sigma_{11} & 0 \\ 0 & \sigma_{22}\end{array}\right]$.

The system is subjected to a constant (isotropic) hydrostatic stress $\sigma_{0}$, to confine the particles. The initial strain and stress are:

$\left[\boldsymbol{\varepsilon}_{0}\right]=\left[\begin{array}{ll}0 & 0 \\ 0 & 0\end{array}\right], \quad\left[\boldsymbol{\sigma}_{0}\right]=\left[\begin{array}{cc}\sigma_{0} & 0 \\ 0 & \sigma_{0}\end{array}\right]$.

The sign convention for strain and stress of [17] is adopted: positive $(+)$ for dilatation/extension and negative $(-)$ for compression/contraction. Therefore, the compressive stress $\sigma_{0}$ is negative. When the top boundary is moved downwards, $\varepsilon_{22}$ (the prescribed strain) will have a negative value whereas $\varepsilon_{11}$, as moving outwards, will be positive.

In general, the strain can be decomposed into an (isotropic) volumetric and a (pure shear) deviatoric part, $\boldsymbol{\varepsilon}=\boldsymbol{\varepsilon}^{V}+\boldsymbol{\varepsilon}^{D}$. The isotropic strain is

$\boldsymbol{\varepsilon}^{V}=\frac{\varepsilon_{11}+\varepsilon_{22}}{2}\left[\begin{array}{ll}1 & 0 \\ 0 & 1\end{array}\right]=\frac{1}{\mathcal{D}} \operatorname{tr}(\boldsymbol{\varepsilon}) \mathbf{I}=\varepsilon^{v} \mathbf{I}$,

with dimension $\mathcal{D}=2$, unit tensor $\mathbf{I}$, and volume change $\operatorname{tr}(\varepsilon)=2 \varepsilon^{v}$, invariant with respect to the coordinate system chosen. Positive and negative $\varepsilon^{v}$ correspond to volume increase and decrease, respectively. Accordingly, the deviatoric strain is:
$\boldsymbol{\varepsilon}^{D}=\boldsymbol{\varepsilon}-\boldsymbol{\varepsilon}^{V}=\frac{\varepsilon_{11}-\varepsilon_{22}}{2}\left[\begin{array}{rr}1 & 0 \\ 0 & -1\end{array}\right]=\gamma \mathbf{I}^{D}$,

where $\gamma=\left(\varepsilon_{11}-\varepsilon_{22}\right) / 2$ is the scalar that describes the pure shear deformation and $\mathbf{I}^{D}$ is the traceless unit-deviator in $2 \mathrm{D}$. The unit-deviator has the eigenvalues, +1 and -1 , with the eigen-directions $\hat{\mathbf{n}}(+1)=\hat{\mathbf{x}}_{1}$ and $\hat{\mathbf{n}}(-1)=\hat{\mathbf{x}}_{2}$, where the hats denote unit vectors.

The same decomposition can be applied to the stress tensor $\sigma=\sigma^{H}+\sigma^{D}$, and leads to the hydrostatic stress

$\boldsymbol{\sigma}^{H}=\frac{\sigma_{11}+\sigma_{22}}{2}\left[\begin{array}{ll}1 & 0 \\ 0 & 1\end{array}\right]=\frac{1}{\mathcal{D}} \operatorname{tr}(\boldsymbol{\sigma}) \mathbf{I}=\sigma^{h} \mathbf{I}$,

and the (pure shear) deviatoric stress

$\boldsymbol{\sigma}^{D}=\sigma-\sigma^{H}=\frac{\sigma_{11}-\sigma_{22}}{2}\left[\begin{array}{rr}1 & 0 \\ 0 & -1\end{array}\right]=\tau \mathbf{I}^{D}$,

with the scalar (pure) shear stress $\tau$. According to their definition, both $\gamma$ and $\tau$ can be positive or negative.

Finally, one additional tensor that describes the difference between the material stiffnesses in $1-$ and $2-$ directions, can be introduced: the structural anisotropy $\mathbf{a}^{D}$ (second order), related to the deviatoric fabric or stiffness/acoustic tensor. Since in the bi-axial system the anisotropy orientation is known, the tensor is fully described by the scalar modulus $A$ :

$\mathbf{a}^{D}=A \mathbf{I}^{D}$.

This leads to three elastic moduli, i.e., bulk-, shear-, and anisotropy-modulus, respectively,

$B=\frac{1}{2}\left[\frac{C_{1111}+C_{2222}+2 \lambda}{2}\right]$,

$G=B-\lambda, \quad$ and

$A=\frac{C_{1111}-C_{2222}}{2}$,

where $C_{1111}, C_{2222}$ and $\lambda=C_{1122}$ are elements of the (rank four) stiffness/acoustic tensor $\mathcal{C}$ of the system. The two constants $\lambda$ and $G$ are the Lamé coefficients of an isotropic material. In a more general constitutive relation of anisotropic elasticity, the tensor $\mathcal{C}$ relates stress and strain increments [17]:

$\delta \boldsymbol{\sigma}=\mathcal{C}: \delta \boldsymbol{\varepsilon}+\delta \boldsymbol{\sigma}^{s}$.

The first term in Eq. (8) is reversible (elastic), while the second contains the stress response due to possibly irreversible changes of structure. Using $B, G$, and $A$, one can directly relate isotropic and deviatoric stress and strain [17]:

$\delta \sigma^{h}=2 B \delta \varepsilon^{v}+A \delta \gamma \quad$ and $\delta \tau=A \delta \varepsilon^{v}+2 G \delta \gamma$.

In summary, for the bi-axial system, the tensors $\varepsilon^{D}, \sigma^{D}$, and $\mathbf{a}^{D}$ can be represented by the scalars $\gamma, \tau$, and $A$, respectively, 
while the orientation is fixed to $\mathbf{I}^{D}$. Change of sign corresponds to reversal of deformation-, stress-, or anisotropydirection.

As special case, the model can describe isotropy, for which the equality $C_{1111}=C_{2222}$ holds, so that $A=0$. Then the second term in the isotropic stress and the first term in the deviatoric stress Eqs. (9) vanish.

In realistic systems, $B$ will increase (decrease) due to isotropic compression (extension) [8] and also $G$ changes due to both isotropic or shear deformation [3]. The models describing this dependence of bulk and shear moduli on strain are in progress, but are neglected here for the sake of simplicity; only constant $B$ and $G$ are considered.

\subsection{Model with evolution of anisotropy}

When there is anisotropy in the system, a positive $A$ in Eqs. (9), in our convention, means that the horizontal stiffness is larger than the vertical ( $A<0$ implies the opposite).

Discrete Element Method simulations [14,15] of an initially isotropic system, with $A_{0}=0$, show that anisotropy builds up to a limit $A^{\max }$ during deformation. It is also observed that the anisotropy varies only due to shear strain and practically not due to volumetric strain. Therefore, the evolution of $A$ is described as:

$\frac{\partial A}{\partial \gamma}=-\beta_{A} \operatorname{sign}\left(A^{\max }\right)\left(A^{\max }-A\right), \quad \frac{\partial A}{\partial \varepsilon^{v}}=0$,

with $A^{\max }=-A^{m} \gamma /|\gamma|=-A^{m} \operatorname{sign}(\gamma)$, see [17], with positive maximal anisotropy, $A^{m}$, i.e., the sign of $A^{\max }$ is determined by the direction of shear. ${ }^{1}$ The rate of anisotropy evolution $\beta_{A}$ determines how fast the anisotropy changes with strain and thus also it approaches (exponentially) its maximum for large $\gamma$. Starting from an isotropic initial configuration, $A_{0}=0$, the growth is linear for small deformations $\gamma$.

\subsection{Non-linear stress evolution}

It is observed from DEM simulations of (horizontal stress controlled) bi-axial deformations $[14,15]$, that the response of the system stress is not linear. For increasing strain the stress increments decrease until the stress saturates at peak (sometimes) and eventually reaches the critical state regime. In $[14,15]$, the evolution equation that leads to saturation, is similar to Eq. (10):

$$
\frac{\partial s_{d}}{\partial \gamma}=\beta_{s} \operatorname{sign}(\gamma)\left(s_{d}^{\max }-s_{d}\right),
$$

\footnotetext{
${ }_{1}$ Assume horizontal compression, which corresponds to $\gamma /|\gamma|<0$, that leads to an increase of horizontal stiffness and thus a positive $A^{\max }$. Compression in vertical direction leads to a negative $A^{\max }$-while tension in horizontal or vertical direction lead to negative and positive $A^{\mathrm{max}}$, respectively.
}

where $s_{d}$ is the stress deviator ratio:

$s_{d}=\frac{\sigma_{11}-\sigma_{22}}{\left(\sigma_{11}+\sigma_{22}\right)}=\frac{\tau}{\sigma^{h}}$,

and $s_{d}^{\max }=-s_{d}^{m} \gamma /|\gamma|=-s_{d}^{m} \operatorname{sign}(\gamma)$, with positive maximum deviatoric stress ratio $s_{d}^{m}$.

Starting from here, a phenomenological extension of the linear model, as described in Eqs. (9), was proposed in [17], leading to the non-linear, incremental constitutive relations:

$$
\begin{aligned}
& \delta \sigma^{h}=2 B \delta \varepsilon^{v}+A S \delta \gamma, \\
& \delta \tau=A \delta \varepsilon^{v}+2 G S \delta \gamma, \text { and } \\
& \delta A=\beta_{A} \operatorname{sign}(\gamma)\left(A^{\max }-A\right) \delta \gamma,
\end{aligned}
$$

where the stress isotropy $S=\left(1-s_{d} / s_{d}^{\max }\right)$ has been introduced. This quantity characterizes the stress-anisotropy in the material, varying between 0 (maximally anisotropic in strain direction), 1 (fully isotropic) up to 2 (maximally anisotropic, perpendicular to the momentary strain increment).

Note that the use of an evolution (or rate-type) equation for the stress allows for irreversibility in the constitutive model due to the terms with $S$ only. This approach is similar to hypoplasticity [13] or GSH [11] and differs from elasto-plastic models. In the former both elastic and plastic strains always coexist [2].

In summary, besides the five local field variables, $\sigma^{h}, \tau$, $\varepsilon^{v}, \gamma$, and $A$, the model has only five material parameters: the bulk and shear moduli $B$ and $G$, the macroscopic coefficient of friction, $s_{d}^{m}$, the rate of anisotropy evolution, $\beta_{A}$, and the maximal anisotropy $A^{m}$. With initial conditions $\sigma_{0}, S_{0}=$ $1-\tau_{0} /\left(\sigma_{0} s_{d}^{\max }\right)$, and $A_{0}$, the model can be integrated from $\varepsilon_{0}^{v}=0$ and $\gamma_{0}=0$.

\section{Results}

In this section the proposed constitutive model will be used to describe the behavior of a granular material when an isobaric axial-symmetric compression (extension) is applied (Sect. 3.1). Vertical compression and extension are then combined in Sect. 3.2 to analyze the response of the material to cyclic loading.

Due to the isobaric stress control, the first equation of the constitutive model, Eq. (13), simplifies to:

$0=2 B \delta \varepsilon^{v}+A S \delta \gamma$.

Different parameters are varied now with the goal to understand their meaning in the model. We chose the range of parameter values roughly referring to soil mechanics and granular materials experiments $[3,12]$. In all examples the confining pressure is $\sigma_{0}=-100 \mathrm{kPa}$, the bulk modulus $B=200 \mathrm{MPa}$ is set constant, whereas for the shear modulus four values are used, $G=25,50,75$, and 
$100 \mathrm{MPa}$, corresponding to the dimensionless ratios $G / B=$ $1 / 8,2 / 8,3 / 8$, and $4 / 8$, respectively.

The samples are initially isotropic (in stress, $S_{0}=1$, and structure, $A_{0}=0$ ) and the maximal anisotropy depends on the bulk modulus such that $A^{m}=B / 2$. The dependence of the model on the anisotropy evolution rate parameter $\beta_{A}$ is tested. For $\beta_{A}=0$ one recovers the special case of isotropy $\left(A=A_{0}=0\right)$. Anisotropic materials with different rate of evolution of anisotropy, display several important features of granular matter behavior. The parameter, $s_{d}^{m}=0.4$ is also chosen from numerical simulations with a reasonable contact coefficient of friction $\mu \approx 0.5[14,15]$.

\subsection{Axial-symmetric isobaric compression}

We study the evolution of anisotropy $A$, deviatoric stress ratio $s_{d}$ and volume strain $2 \varepsilon^{v}$, for vertical compression, (i.e., for positive $\gamma$ ) with constant anisotropy evolution rate $\beta_{A}$ and different shear moduli $G$. Since the evolution of $A$, see Eq. (15), is not affected by $G$, we do not show it here, but refer to Fig. 3a below. For the chosen set of parameters, the anisotropy reaches its (negative) extreme value within about $0.1 \%$ of strain, where the sign indicates the fact that the stiffness in vertical (compression) direction is larger than in horizontal (extension) direction.

The deviatoric stress (positive, normalized by the constant, negative confining pressure, in order to keep its sign), is plotted in Fig. $2 \mathrm{a}$ as the stress ratio $-s_{d}=-\tau / \sigma_{0}$. It increases linearly, with slope $2 G / \sigma_{0}$, to positive values and saturates at $s_{d}^{m}$. Positive $-\tau / \sigma_{0}$ means that the vertical (compressive) stress magnitude is larger than the horizontal (compressive) stress-both negative in sign, due to our convention. In Fig. $2 b$, the volumetric strain, $2 \varepsilon^{v}$, increases and saturates at values between $\gamma=7 \times 10^{-4}$ and $0.5 \times 10^{-4}$, for different $G$. Since both $B$ and $A$ in Eq. (16) do not depend on $G$, this dilatancy is only due to the different evolution of the stress isotropy $S$ with strain, as explained below.

In Fig. 3, the dependence of the model on different rates of anisotropy evolution, $\beta_{A}$, is displayed. The predictions for $A,-\tau / \sigma_{0}$, and $2 \varepsilon^{v}$ are plotted for fixed shear modulus $G=25 \mathrm{MPa}$ and different $\beta_{A}$. From $A\left(\beta_{A}, \gamma\right)$ as displayed in Fig. 3a, see Eq. (10), one observes that for the extremely large $\beta_{A}=10^{6}$, one practically has instantaneously the maximum $A=A^{\max }$. For decreasing $\beta_{A}$, the initial slope $-\beta_{A} A^{m} / 2 B=-\beta_{A} / 4$, decreases, while all curves saturate at $A^{\mathrm{max}}=-B / 2$. The isotropic case of minimal $\beta_{A}=0$, is clearly distinct from the other cases, since one has constant $A=0$.

The stress curves in Fig. 3b initially increase with slope $2 G / \sigma_{0}$ but, for very small strain-due to the evolution of $A$-become "softer": the larger $\beta_{A}$, the stronger the deviation from the initial slope. They finally saturate at $-\tau / \sigma_{0}=$ $s_{d}^{m}=0.4$, as prescribed, within similar strains $\gamma \approx 0.7 \%$.
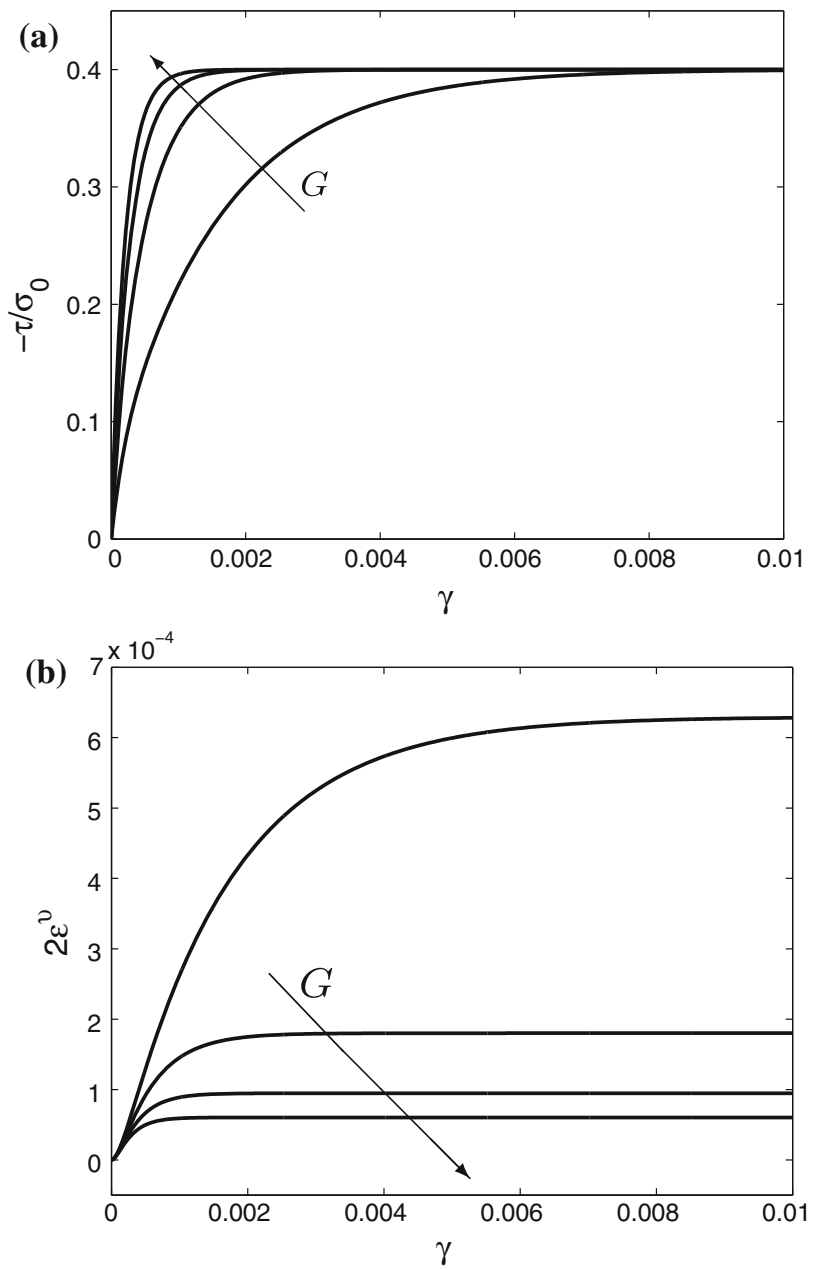

Fig. 2 (a) Deviatoric stress ratio and (b) volumetric strain, during isobaric axial-symmetric compression, as function of the deviatoric strain, $\gamma$, for the parameters $\sigma_{0}=-100 \mathrm{kPa}, B=200 \mathrm{MPa}, s_{d}^{m}=0.4$, for evolving $A$, with $\beta_{A}=6000, A^{m}=B / 2$, and for different shear moduli, $G=25,50,75$, and $100 \mathrm{MPa}$, increasing right-to-left (a) or top-tobottom (b)

With other words, the behavior always starts isotropic, since $A_{0}=0$, that is, for all $\beta_{A}$, the curve for $\beta_{A}=0$ is valid when $\gamma \rightarrow 0$. The stress increase is then slower for more anisotropic materials, since negative $A$, together with positive $\delta \varepsilon^{v}$ in Eq. (14), works against the stress saturation. Finally, when $A$ has approached its maximum-faster than the stress- the stress saturation becomes independent of $\beta_{A}$ again.

The volumetric strain, see Fig. $3 c$, increases with deviatoric strain according to Eq. (16) and saturates at increasing $\varepsilon^{v}$, for increasing $\beta_{A}$. Again $\beta_{A}=0$ represents the volume conserving limit case (in contrast to experimental evidence [10]) and all the curves are tangent in the origin to the corresponding line $\varepsilon^{v}=0$. This is due to Eq. (16), where $A=0$, for isobaric axial-symmetric compression, leads to $\delta \varepsilon^{v}=-A S \delta \gamma / 2 B=0$. With applied pure shear, volumetric strain can not exist without anisotropy. Nevertheless, the iso- 

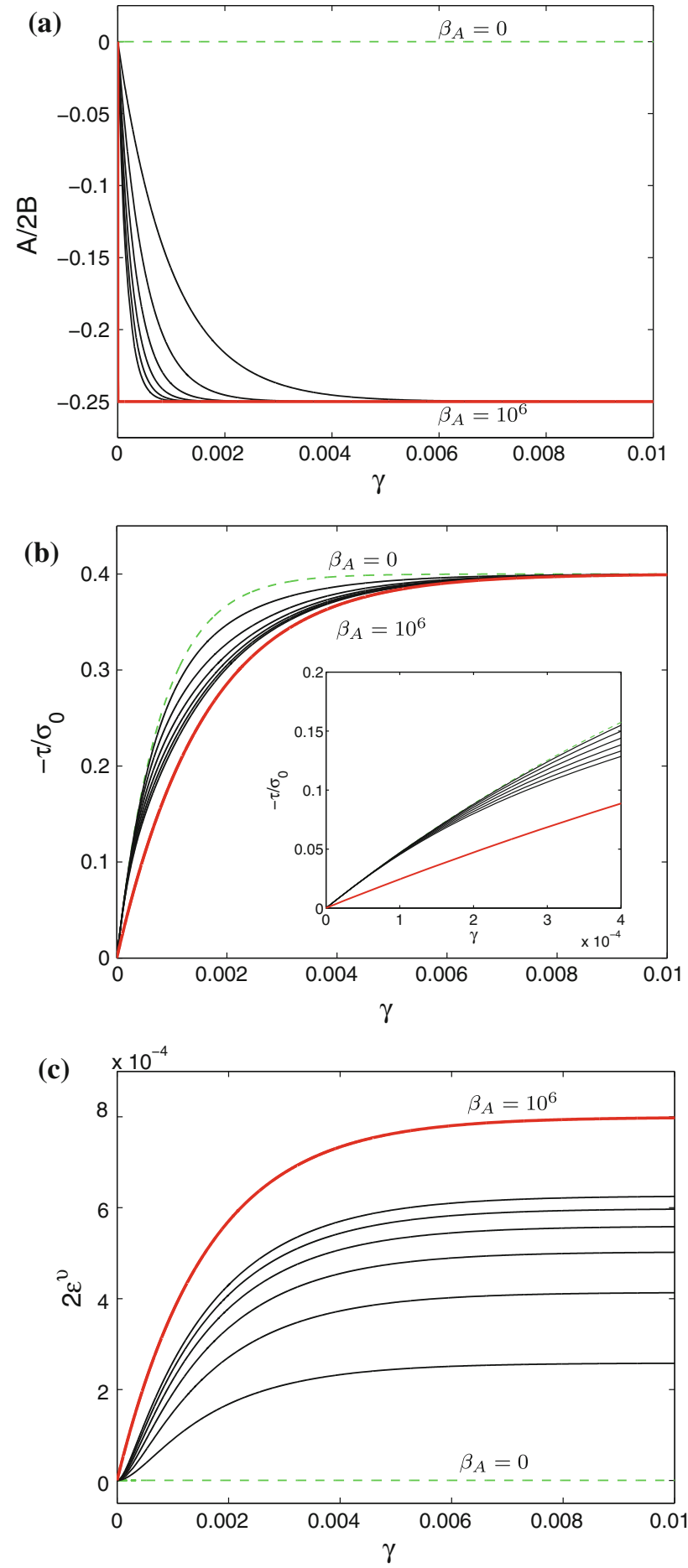

Fig. 3 (a) Anisotropy, (b) deviatoric stress ratio, and (c) volumetric strain during isobaric axial-symmetric deformation, as function of deviatoric strain, $\gamma$, with $\sigma_{0}=-100 \mathrm{kPa}, B=200 \mathrm{MPa}$ and $G=25 \mathrm{MPa}$, for evolving $A$, with $A^{m}=B / 2$ and varying $\beta_{A}$, increasing from 0 to 6000 in steps of 1000, from top to bottom in panels (a) and (b), and from bottom to top in panel (c). The inset in (b) is a zoom into the small strain response. The green-dashed and red-solid lines represent the extreme cases of isotropy $\beta_{A}=0,(A=0)$ and constant, instantaneous maximal anisotropy, $\beta_{A}=10^{6},\left(A=A^{\mathrm{max}}\right)$, respectively. (Color figure online) tropic case $A_{0}=0$ can be a proper description of the incremental response of an initially isotropic granular material, when very small strains are applied (consistent with experimental observations).

Similar results (besides different signs) are obtained when an axially symmetric vertical extension with constant confining pressure is applied.

\subsection{Strain reversal}

Now vertical compression and extension are combined, resembling cyclic loading. The path is strain-controlled, that is the strain increment is reversed after a certain shear strain is accumulated. We start with compression until about $1 \%$ of vertical integrated strain, $\varepsilon_{22} \simeq 0.01$, is reached, ensuring the system to be in the well established critical state flow regime (for large $\beta_{A}$ ). After reversal, vertical extension is carried on until it also reaches $1 \%$, relative to the original configuration. At this point the increment is reversed again and a new compression-extension cycle starts.

In particular, we want to understand how the rate of anisotropy evolution $\beta_{A}$ influences the cyclic loading path. For fixed shear (and bulk) modulus, we compare the behavior for two different values of $\beta_{A}$. Figs. $4 \mathrm{a}, \mathrm{c}, \mathrm{e}, \mathrm{g}$ and $4 \mathrm{~b}, \mathrm{~d}, \mathrm{f}, \mathrm{h}$ show the system properties as functions of the deviatoric strain, $\gamma$, for anisotropy rates $\beta_{A}=2000$ and 400, respectively. For both anisotropy $(a, b)$ and stress-ratio $(c, d)$, except for the first loading, this relation consists of hysteresis loops of constant width as consecutive load-unload cycles are applied. This hysteresis produces an accumulation of both isotropic and deviatoric strain, positive for large $\beta_{A}$ and negative for small $\beta_{A}$, see Figs. $4 \mathrm{e}$,f. Figures $4 \mathrm{c}$ and $4 \mathrm{~d}$ show that the deviatoric stress increases due to compression, until load reversal (extension) and decreases to negative values until the next reversal. Under load reversal, the corresponding stress response is realized with identical loading and un-loading stiffnesses. In agreement with Fig. 3b, anisotropy decreases (increases) faster for larger $\beta_{A}$, whereas the stress ratio $\tau / \sigma_{0}$ approaches its maximum somewhat slower for lower $\beta_{A}$, due to the opposite signs of the two terms on the r.h.s. in Eq. (14).

In Figs. $4 \mathrm{e}$ and $4 \mathrm{f}$, the accumulation of small permanent deformations, after each cycle, both isotropic and deviatoric, are displayed. Overall, the ratcheting leads to an increase (decrease) of volume in each cycle for large (small) $\beta_{A}$, respectively. The sign-reversal of the anisotropy modulus $A$ during each half-cycle is responsible for the sign of the volumetric strain. This comes directly from the analysis of Eq. (16): the stress isotropy $S$ can only be positive and $A$ changes sign with increasing deviatoric strain $\gamma$, after each reversal. The volumetric strain accumulates monotonically following the behavior of $A$. Interestingly, $\beta_{A}$, the rate of change of $A$, controls the net volume change. The original 

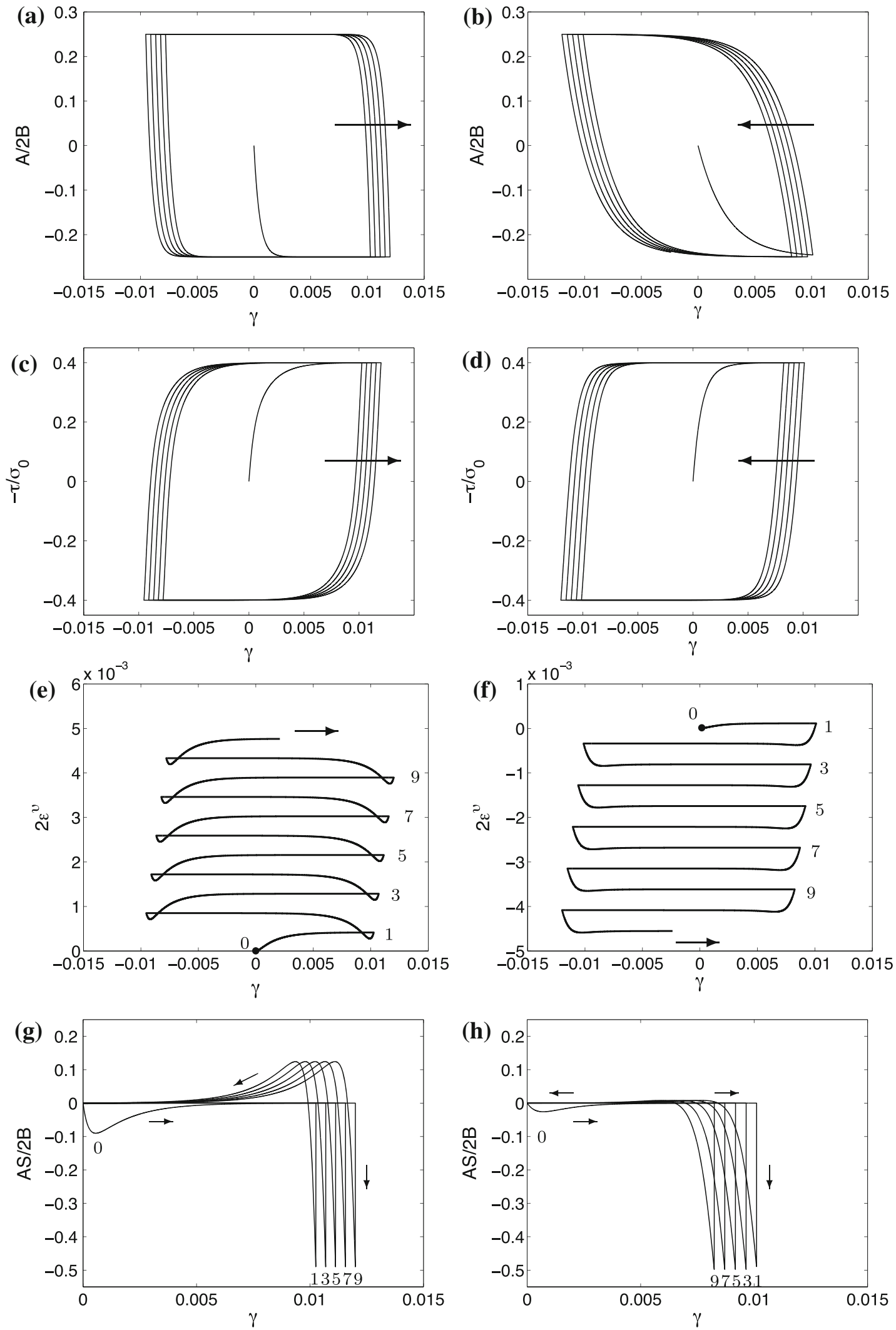

Fig. 4 Anisotropy, $A / 2 B(\mathbf{a}, \mathbf{b})$, deviatoric stress ratio, $-\tau / \sigma_{0}(\mathbf{c}, \mathbf{d})$, volumetric strain, $2 \varepsilon^{v}(\mathbf{e}, \mathbf{f})$, and the contractancy/dilatancy ratio $A S / 2 B$ $(\mathbf{g}, \mathbf{h})$, during isobaric axial-symmetric deformation and cyclic loading,

with $\sigma_{0}=-100 \mathrm{kPa}, G=25 \mathrm{MPa}$ and $B=200 \mathrm{MPa}$, for evolving $A, A^{m}=B / 2, \beta^{A}=2000$ (Left) and $\beta^{A}=400$ (Right) 
reason for this behavior becomes clear looking at Figs. $4 \mathrm{~g}$ and $4 \mathrm{~h}$, where the variation of the contractancy/dilatancy ratio $A S / 2 B$ (during initial loading (0) and the odd reversal points $(1,3,5,7,9))$ is shown for the two different $\beta_{A}$. At the beginning $(0)$, the quantity becomes always negative due to the initial decrease of $A$ : that is, the system always shows initial dilatancy, see path $0-1$ in Figs. $4 \mathrm{e}$ and 4f. Only after the first reversal, the influence of $\beta_{A}$ on dilatancy/compactancy shows up. This initial part of the cyclic loading corresponds to what is discussed in Fig. 3. The integration of $A S / 2 B$ over $\gamma$ leads to increasing $\varepsilon^{v}$ in the first case (g) and decreasing $\varepsilon^{v}$ in the second (h). Rapid changes of the anisotropy modulus $A$, corresponding to large $\beta_{A}$, lead to dilation, whereas slow changes of $A$ lead to compaction.

Besides the trivial case of anisotropy rate $\beta_{A}=0$, the analysis leads to the existence of a second critical value $\beta_{A}^{c}$ such that there is no volume change in the material, as shown in Fig. 5a. The second material parameter in Eq. (10), the maximal anisotropy $A^{m}$, also influences ratcheting. For all $A^{m}$ studied, the volume change per cycle rapidly drops and then increases with $\beta_{A}$, reaching larger values for larger $A^{m}$. The critical $\beta_{A}^{c}$, corresponding to no volume change, decreases with $A^{m}$ increasing. The amount of strain accumulation per cycle, $\Delta 2 \varepsilon^{v}$, and $\beta_{A}^{c}$ also depend on the shear modulus $G$, see Fig. 5b. In fact, larger $G$ leads to a faster increase of the stress deviator ratio $s_{d}$, that is to a faster decrease of the stress isotropy $S$ in Eq. (16). Moreover, the critical value $\beta_{A}^{c}$ increases when the shear modulus increases.

The behavior reported in Figs. $4 \mathrm{e}$ and $4 \mathrm{f}$ is qualitatively in agreement with physical experiments. Strain accumulation appears, when a granular sample is subjected to shear stress reversals in a triaxial cell with constant radial stress [21] or in a torsional resonant column with constant mean-stress [4]. Interestingly, the behavior of the material is shown to be amplitude dependent in [21]. The model is able to reproduce such a dependence, the strain accumulation vanishing for very small amplitude (data not shown). The accumulation per cycle, that is constant in the present model, see Figs. 4e and $4 \mathrm{f}$, in our opinion will become cycle- and strain-dependent when evolution laws for $B$ and $G$ are considered. An accurate comparison with experimental and numerical data is subject of a future study.

\section{Summary and conclusion}

In the bi-axial system — where the eigen-vectors of all tensors are either horizontal or vertical-a new constitutive model, as inspired by DEM simulations [14,15], is presented in Eqs. (13), (14), and (15). It involves incremental evolution equations for the hydrostatic and deviatoric stresses and for the single (structural) anisotropy modulus that varies differently from the stress-anisotropy during deviatoric deforma-
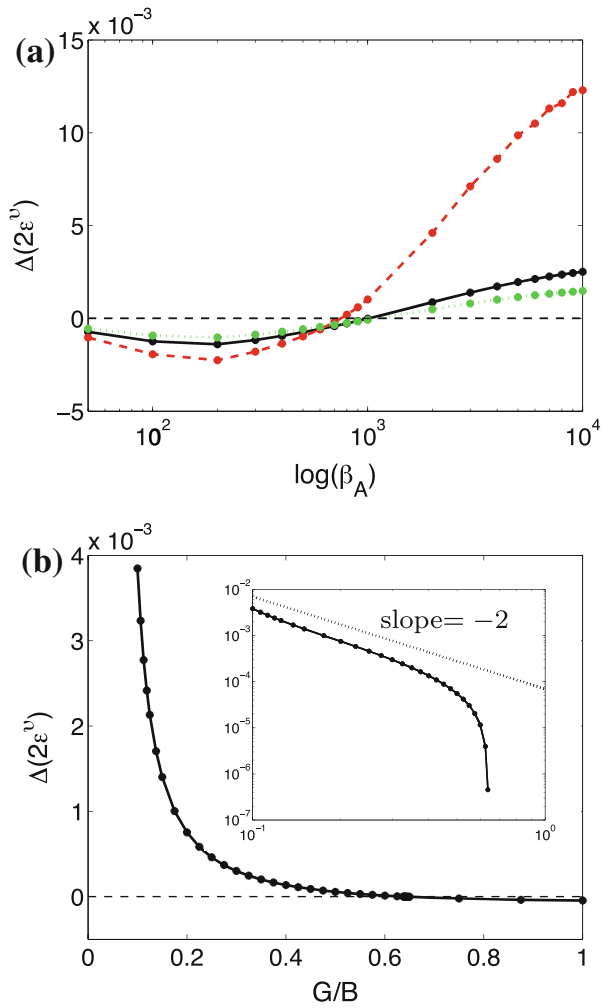

Fig. 5 Strain accumulation per cycle $\Delta\left(2 \varepsilon^{v}\right)$ with $\sigma_{0}=-100 \mathrm{kPa}$ and $B=200 \mathrm{MPa}$ for evolving $A$. In panel (a) $\Delta\left(2 \varepsilon^{v}\right)$ is plotted against $\log \left(\beta^{A}\right)$ with $A^{m}=2 B / 3$ (red dashed line), $A^{m}=B / 2$ (black solid line), $A^{m}=2 B / 5$ (green dotted line) and fixed $G=25 \mathrm{MPa}$. In panel (b) $\Delta\left(2 \varepsilon^{v}\right)$ is plotted against $G / B$, with fixed $\beta_{A}=6000$; in the inset the same quantities are plotted in logarithmic scale. (Color figure online)

tions of the system and thus represents a history/memory parameter [17]. The five local field variables are $\sigma^{h}, \tau, \varepsilon^{v}, \gamma$, and $A$.

The model involves only three moduli: the classical bulk modulus, $B$, the shear modulus, $G$, and the anisotropy modulus, $A$, whose sign indicates the direction of anisotropy in the present formulation. Due to the anisotropy, $A$, the model involves a cross coupling of the two types of strains and stresses, namely isotropic and shear (deviatoric). As opposed to isotropic materials, shear strain can cause e.g. dilation and hence compressive stresses. Similarly, a purely volumetric strain can cause shear stresses and thus shear deformation in the system. As main hypothesis, the anisotropy evolution is controlled by the anisotropy rate $\beta_{A}$ and by deviatoric strain, $\gamma$, but not (directly) by stress.

The model also leads to a critical state regime, where the volume, the stresses, and the anisotropy modulus do not change anymore. The critical state is described by the maximal anisotropy $A^{m}$ and the maximal deviatoric stress ratio $s_{d}^{m}$, equivalent to a macroscopic friction coefficient [16].

To better understand the model, a series of simulations has been performed for special cases. For very small strains, lin- 
ear relations between stresses and strains are observed, while for larger strains the non-linear behavior sets in with a particular cross-coupling between isotropic and deviatoric components through both stress-ratio, $s_{d}$, and structure-anisotropy, $A$-leading to non-linear response at load-reversal. Dilation or compaction after large amplitude load reversal are related to fast or slow evolution of the anisotropy, $A$, respectively.

Comparison with DEM simulations is in progress. The next step is the formulation of the model for arbitrary orientations of the stress-, strain- and anisotropy-tensors, but keeping the number of material parameters fixed. This will eventually allow, e.g., a finite element method implementation, in order to study arbitrary boundary conditions other than homogeneous bi-axial systems. Furthermore, the model will be generalized to three dimensions in the spirit of [17], where (at least) one more additional anisotropy parameter (tensor) is expected to be present for arbitrary deformation histories.

Acknowledgments This work is dedicated to late Prof. I. Vardoulakis and his inspiring publications. Helpful discussions with J. Goddard, D. Krijgsman, M. Liu, S. McNamara, E. S. Perdahciog̃lu, A. Singh, S. Srivastava, H. Steeb, J. Sun and S. Sundaresan are gratefully acknowledged. The work was financially supported by an NWO-STW VICI grant.

Open Access This article is distributed under the terms of the Creative Commons Attribution Noncommercial License which permits any noncommercial use, distribution, and reproduction in any medium, provided the original author(s) and source are credited.

\section{References}

1. Alonso-Marroquin, F., Herrmann, H.J.: Ratcheting of granular materials. Phys. Rev. Lett 92(5), 054301 (2004)

2. Alonso-Marroquin, F., Luding, S., Herrmann, H.J., Vardoulakis, I.: Role of anisotropy in the elastoplastic response of a polygonal packing. Phys. Rev. E 71, 0513404 (2005)

3. Chen, Y., Ishibashi, I., Jenkins, J.: Dynamic shear modulus and fabric: part I, depositional and induced anisotropy. Géotechnique 1(38), 25-32 (1988)

4. Chen, Y., Ishibashi, I., Jenkins, J.: Dynamic shear modulus and fabric: part II, stress reversal. Géotechnique 1(38), 33-37 (1988)

5. de Gennes, P.G.: Granular matter: a tentative view. Rev. Mod. Phys. 71(2), 374-382 (1999)

6. Geng, L., Reydellet, G., Clément, E., Behringer, R.P.: Green's function measurements in 2D granular materials. Physica D 182, 274 303 (2003)

7. Goddard, J.: Granular hypoplasticity with Cosserat effects. In: Goddard, J., Giovine, P., Jenkins, J.T. (eds.) IUTAM-ISIMM Symposium on Mathematical Modeling and Physical Instances of Granular Flows, AIP, 14-18 September 2009, pp. 323-332. Reggio Calabria (Italy) (2010)
8. Göncü, F., Duran, O., Luding, S.: Constitutive relations for the isotropic deformation of frictionless packings of polydisperse spheres. C. R. Mecanique 338(10-11), 570-586 (2010)

9. Jaeger, H.M., Nagel, S.R., Behringer, R.P.: Granular solids, liquids, and gases. Rev. Mod. Phys 68(4), 1259-1273 (1996)

10. Jenkins, J., Cundall, P., Ishibashi, I.: Micromechanical modeling of granular materials with the assistance of experiments and numerical simulations. In: Biarez, J., Gourves, M. (eds.) Powders and Grains, pp. 257-264. Balkema, Rotterdam (1989)

11. Jiang, Y., Liu, M.: Granular solid hydrodynamics. Granul. Matter 11(3), 139-156 (2009)

12. Khidas, Y., Jia, X.: Anisotropic nonlinear elasticity in a spherical-bead pack: influence of the fabric anisotropy. Phys. Rev. E 81, 21303 (2010)

13. Kolymbas, D.: An outline of hypoplasticity. Arch. App. Mech. 61, 143-154 (1991)

14. Luding, S.: Micro-macro models for anisotropic granular media. In: Vermeer, P.A., Ehlers, W., Herrmann, H.J., Ramm E. (eds.) Modelling of Cohesive-Frictional Materials, pp. 195-206. Balkema, Leiden, The Netherlands (2004)

15. Luding, S.: Anisotropy in cohesive, frictional granular media. J. Phys.: Condens. Matter 17, S2623-S2640 (2005)

16. Luding, S., Alonso-Marroquin, F.: The critical-state yield stress (termination locus) of adhesive powders from a single numerical experimen. Granul. Matter 13(2), 109-119 (2011)

17. Luding, S., Perdahcioglu, S.: A local constitutive model with anisotropy for various homogeneous 2D biaxial deformation modes. CIT, doi:10.1002/cite.201000180 (2011)

18. Muhlhaus, H., Moresi, L., Gross, L., Grotowski, J.: The influence of non-coaxiality on shear banding in viscous-plastic materials. Granul. Matter 12, 229-238 (2010)

19. Sun, J., Sundaresan, S.: A plasticity model with microstructure evolution for quasi-static granular flows. In: Goddard, J., Giovine, P., Jenkins, J.T. (eds.) IUTAM-ISIMM Symposium on Mathematical Modeling and Physical Instances of Granular Flows, 14-18 September 2009, AIP, pp. 280-289. Reggio Calabria (Italy) (2010)

20. Sun, J., Sundaresan, S.: A plasticity model with microstructure evolution for quasi-static granular flows. J. Fluid Mech. (2010 submitted)

21. Tatsuoka, F., Ishihara, K.: Drained deformation of sand under cyclic stresses reversing direction. Soils. Found. 3(14), 51-65 (1974)

22. Tejchman, J., Bauer, E., Wu, W.: Effect of fabric anisotropy on shear localization in sand during plane strain compression. Acta Mech. 189, 23-51 (2007)

23. Vardoulakis, I., Frantziskonis, G.: Micro-structure in kinematichardening plasticity. Eur. J. Mech. A Solids 11(4), 467 (1992)

24. Vardoulakis, I., Sulem, J.: Bifurcation Analysis in Geomechanics. Chapman and Hall, London (1995)

25. Zhang, J., Majmudar, T.S., Tordesillas, A., Behringer, R.P.: Statistical properties of a 2D granular material subjected to cyclic shear. Granul. Matter 12, 159-172 (2010) 